\title{
O USO DA BIXINA COMO ANTIOXIDANTE NATURAL EM DIETAS DE FRANGOS DE CORTE FORMULADAS COM ÓLEO DE SOJA FRESCO OU OXIDADO
}

(Bixin as natural antioxidant in broilers diet containing fresh or oxidized soybean oil)

Fabiana Golin Golin Luiggi ${ }^{1}$, Peterson Dante Gavasso Pacheco ${ }^{1}$, Aline Mondini Calil Racanicci ${ }^{2}$, Elisângela de Souza Miranda Muynarsk ${ }^{3}$, Marcia Maria Pereira Sartori ${ }^{4}$, José Roberto Sartori ${ }^{1}$

${ }^{1}$ Faculdade de Medicina Veterinária e Zootecnia - UNESP/Botucatu. Departamento de Melhoramento e Nutrição Animal, Botucatu, São Paulo, Brasil, ${ }^{2}$ Faculdade de Agronomia e Medicina Veterinária, Universidade de Brasília, Departamento de Zootecnia, Brasília, Distrito Federal, Brasil, ${ }^{3}$ Instituto de Biociências - UNESP/Botucatu. Departamento de Microbiologia e Imunologia, Botucatu, São Paulo, Brasil, ${ }^{4}$ Faculdade de Ciências Agronômicas - UNESP/Botucatu. Departamento de Produção e Melhoramento Vegetal, Botucatu, São Paulo, Brasil

Corresponding author: luiggi.fabi@gmail.com

RESUMO: A adição de óleos e gorduras na dieta de frangos de corte é bastante comum, uma vez que esses ingredientes são boas fontes de energia. No entanto, estes ingredientes sofrem facilmente deterioração através da oxidação lipídica, e o consumo de alimentos ou ingredientes oxidados pode prejudicar o desempenho dos frangos de corte. Com o objetivo de avaliar as propriedades antioxidantes da bixina adicionada à dieta de frangos de corte formulada com óleo de soja fresco ou oxidado e o efeito do óleo de soja oxidado na dieta das aves, foi realizado um experimento com 1000 frangos de corte, machos, de 1 dia de idade, Ross 308 . O delineamento experimental foi inteiramente casualizado, em esquema fatorial $3 \times 2$ + 2 controles positivos, totalizando oito tratamentos com cinco repetições de 25 aves cada. Os tratamentos foram 1) Dieta basal (DB) + óleo de soja fresco (OF); 2) $\mathrm{DB}+$ óleo de soja oxidado (OO); 3 ) $\mathrm{DB}+\mathrm{OF}+0,05 \%$ de bixina; 4) $\mathrm{DB}+\mathrm{OO}+$ $0,05 \%$ de bixina; 5) $\mathrm{DB}+\mathrm{FO}+0,10 \%$ de bixina; 6$) \mathrm{DB}+\mathrm{OO}+0,10 \%$ de bixina; 7 ) $\mathrm{DB}+\mathrm{FO}+\mathrm{BHT}$ (controle positivo 1); e 8) DB + OO + BHT (controle positivo 2). Apesar de terem sido observados efeitos antioxidantes na carne de peito e coxa em alguns momentos, a bixina não pode ser recomendada como uma alternativa natural ao BHT. A inclusão de bixina na dieta dos frangos de corte melhorou a conversão alimentar e reduziu a gordura abdominal, contudo, o uso desse aditivo e de outros carotenóides na dieta dos frangos de corte exige cautela, uma vez que o excesso pode apresentar efeito negativo na qualidade da carne, aumentando a oxidação lipídica devido ao efeito pró-oxidante, e reduzindo a shelf-life. O uso de óleo de soja oxidado degomado prejudica a conversão alimentar e aumenta a deposição de gordura abdominal em frangos de corte.

Palavras-chave: Aditivo; qualidade de carne; oxidação lipídica; urucum.

ABSTRACT: The addition of oils and fats in the diet of broilers is quite common, since these ingredients are good sources of energy. However, these ingredients easily suffer deterioration through lipid oxidation, and the consumption of oxidized foods or ingredients may impair the performance of broilers. In order to evaluate the antioxidant properties of bixin added to the diet of broilers formulated with fresh or oxidized soybean oil and the effect of the oxidized soybean oil on broiler diets, an experiment was carried out with 1000 one-day-old male chicks, Ross 308 . The experimental design was completely randomized, arranged in factorial $3 \times 2+2$ 
positive controls, totalizing eight treatments with five replicates of 25 birds each. The treatments were 1) Basal diet (BD) + fresh soybean oil (FSO); 2) BD + oxidized soybean oil (OSO); 3) BD + FSO + 0.05\% bixin; 4) BD + OSO + 0.05\% bixin; 5) BD $+\mathrm{FSO}+0.10 \%$ bixin; 6$) \mathrm{BD}+\mathrm{OSO}+0.10 \%$ bixin; 7$) \mathrm{BD}+\mathrm{FSO}+\mathrm{BHT}$ (positive control 1); and 8) BD + OSO + BHT (positive control 2). Although antioxidant effects have been observed in breast and thigh meat at some times, bixin can not be recommended as a natural alternative to $\mathrm{BHT}$. The inclusion of bixin in broilers diet improved feed conversion ratio and reduced abdominal fat, however, the use of this additive and other carotenoids in the broilers diet requires caution, since the excess may have a negative effect on the quality of the meat, increasing the lipid oxidation due to the pro-oxidant effect, and reducing the shelf-life. The use of degummed oxidized soybean oil impairs feed conversion ratio and increases the deposition of abdominal fat in broilers.

Keywords: Additive; annatto; lipid oxidation; meat quality. 


\section{INTRODUÇÃO}

\section{O rápido}

desenvolvimento apresentado pelas linhagens de frangos de corte atuais exige alta demanda de energia na dieta, o que, por sua vez, motiva o uso de óleos e gorduras nas rações, ingredientes com alto teor calórico (Macari et al., 2002). No entanto, estes ingredientes apresentam reações espontâneas com oxigênio e sofrem deterioração através da oxidação ou processos de peroxidação lipídica, que são a principal causa de perda de qualidade alimentar, alterando seu sabor, odor, cor, textura e valores nutricionais (Scott, 1982).

O consumo de alimentos ou ingredientes oxidados pode prejudicar o desempenho dos frangos (Lin et al., 1989; Sheehy et al., 1994), devido ao reduzido conteúdo energético dos lipídios oxidados e à presença de altas quantidades de compostos rançosos considerados tóxicos e prejudiciais à absorção de outros nutrientes, como vitaminas lipossolúveis e alguns ácidos graxos essenciais. Os autores explicam que, dependendo da origem do alimento e das condições a que foi submetido durante a oxidação (temperatura e duração do aquecimento, adição de oxigênio e catalisadores, e atividade de água), pode ser formada grande variedade de compostos de ranço quimicamente diferentes. Esses compostos provocam danos às células epiteliais do intestino e ao fígado, prejudicando a absorção e o aproveitamento de nutrientes e energia (Cabel e Waldroup, 1988; Engberg et al., 1996).

Para evitar o processo de oxidação e reduzir os danos causados pelo consumo de alimentos oxidados, é comum o uso de produtos com propriedades antioxidantes na alimentação animal e na indústria alimentícia. Antioxidantes sintéticos, como o hidroxianisolbutilado (BHA) e o hidroxitoluenobutilado (BHT) têm sido frequentemente utilizados.
No entanto, relatos de um possível efeito carcinogênico dessas substâncias aumentam 0 interesse em encontrar alternativas naturais (Zheng e Wand, 2001).

A bixina, um carotenóide presente em grande quantidade em sementes de urucum, possui propriedades antioxidantes que foram confirmadas em diversos estudos (Silva et al., 2001; Lima et al., 2003; Garcia et al., 2012). No entanto, ainda não se sabe se a bixina pode atuar como um antioxidante natural quando fornecida aos frangos de corte via dieta, ou, ainda, se possui capacidade de proteger as aves dos danos causados pela ingestão de alimentos oxidados.

O objetivo deste estudo foi avaliar as propriedades antioxidantes da bixina proveniente do extrato de urucum, adicionada à dieta de frangos de corte formulada com óleo de soja fresco ou oxidado, bem como os efeitos negativos do fornecimento de óleo de soja oxidado na dieta dos animais sobre 0 desempenho.

\section{MATERIAL E MÉTODOS}

Os frangos de corte foram criados no galpão experimental da Agência Paulista de Tecnologia dos Agronegócios - APTA, Pólo Regional do Centro Oeste, Unidade de Pesquisa e Desenvolvimento, Brotas - SP, e abatidos no Abatedouro Experimental da Faculdade de Medicina Veterinária e Zootecnia - UNESP, Botucatu-SP.

Foram utilizados 1.000 frangos machos Ross 308 de um dia de idade vacinados no incubatório contra doenças de Marek, Gumboro, Bronquite e New Castle. As aves foram alojadas em 40 boxes ( 25 aves/boxe) com cama de maravalha, comedouros tubulares e bebedouros tipo nipple. A temperatura e ventilação foram controladas com o uso de cortinas e ventiladores.

O delineamento experimental foi inteiramente casualizado, em esquema 
fatorial $3 \times 2+2$ controles positivos, totalizando oito tratamentos com cinco repetições de 25 aves cada. O esquema fatorial contou com dois tipos de óleo de soja utilizados, fresco e oxidado, e com três níveis de inclusão de extrato oleoso de urucum (tratamentos 1 a 6). Como controle positivo foram adicionados dois tratamentos contendo $\mathrm{O}$ antioxidante sintético BHT, com óleo fresco ou oxidado, definidos como controle positivo, posteriormente comparados com os tratamentos do esquema fatorial (tratamentos 7 e 8).

Dessa forma, os tratamentos
1) Dieta basal (DB) + óleo de soja fresco (OF);

2) $\mathrm{DB}+$ óleo de soja oxidado (OO);

3) $\mathrm{DB}+\mathrm{OF}+0,05 \%$ de bixina;

4) $\mathrm{DB}+\mathrm{OO}+0,05 \%$ de bixina;

5) $\mathrm{DB}+\mathrm{FO}+0,10 \%$ de bixina;

6) $\mathrm{DB}+\mathrm{OO}+0,10 \%$ de bixina;

7) $\mathrm{DB}+\mathrm{FO}+\mathrm{BHT}$ (controle positivo 1);

8) $\mathrm{DB}+\mathrm{OO}+\mathrm{BHT}$ (controle positivo 2).

As dietas basais foram isoproteicas e isocalóricas, formuladas de acordo com as recomendações nutricionais de Rostagno et al. (2011), sem adição de qualquer antioxidante sintético (Tabela 1), exceto para os tratamentos controle positivo.

Tabela 1. Composição centesimal e valores nutricionais calculados das rações basais.

\begin{tabular}{|c|c|c|c|c|}
\hline Ingredientes & $\begin{array}{l}\text { Pré-inicial } \\
\text { (1-7 dias) }\end{array}$ & $\begin{array}{c}\text { Inicial } \\
\text { (8-21 dias) }\end{array}$ & $\begin{array}{c}\text { Crescimento } \\
\text { (22-35 dias) }\end{array}$ & $\begin{array}{c}\text { Final } \\
\text { (36-42 dias) }\end{array}$ \\
\hline Milho, grão & 51,11 & 56,14 & 59,52 & 63,76 \\
\hline Soja, farelo & 39,66 & 36,28 & 32,68 & 28,76 \\
\hline Fosfato bicálcico & 1,92 & 1,56 & 1,35 & 1,13 \\
\hline Calcário calcítico & 0,91 & 0,96 & 0,89 & 0,80 \\
\hline Sal comum & 0,51 & 0,49 & 0,46 & 0,45 \\
\hline Soja, óleo bruto & 3,57 & 3,57 & 4,35 & 4,28 \\
\hline Treonina & 0,11 & 0,08 & 0,07 & 0,07 \\
\hline DL - Metionina & 0,23 & 0,20 & 0,19 & 0,18 \\
\hline L - Lisina & 0,27 & 0,23 & 0,23 & 0,26 \\
\hline Inerte(caulim) & 1,39 & 0,19 & 0,00 & 0,00 \\
\hline Premix vitamínico mineral $^{1}$ & 0,33 & 0,33 & 0,33 & 0,33 \\
\hline TOTAL & 100,00 & 100,00 & 100,00 & 100,00 \\
\hline \multicolumn{5}{|l|}{ Composição calculada } \\
\hline EMAn $(\mathrm{kcal} / \mathrm{kg})$ & 2.960 & 3.050 & 3.150 & 3.200 \\
\hline Proteína Bruta (\%) & 22,40 & 21,20 & 19,80 & 18.41 \\
\hline Cálcio (\%) & 0,92 & 0,85 & 0,76 & 0.67 \\
\hline Fósforo disponível (\%) & 0,47 & 0,40 & 0,36 & 0.31 \\
\hline Sódio (\%) & 0,22 & 0,21 & 0,20 & 0.20 \\
\hline \multicolumn{5}{|l|}{ Aminoácidos digestíveis } \\
\hline Lisina $(\%)$ & 1,33 & 1,22 & 1,13 & 1,06 \\
\hline Metionina (\%) & 0,52 & 0,48 & 0,46 & 0,43 \\
\hline Metionina + Cistina (\%) & 0,82 & 0,77 & 0,73 & 0,68 \\
\hline Triptofano (\%) & 0,26 & 0,24 & 0,22 & 0,20 \\
\hline Treonina (\%) & 0,86 & 0,80 & 0,74 & 0,69 \\
\hline Arginina (\%) & 1,43 & 1,34 & 1,24 & 1,13 \\
\hline \multicolumn{5}{|c|}{ 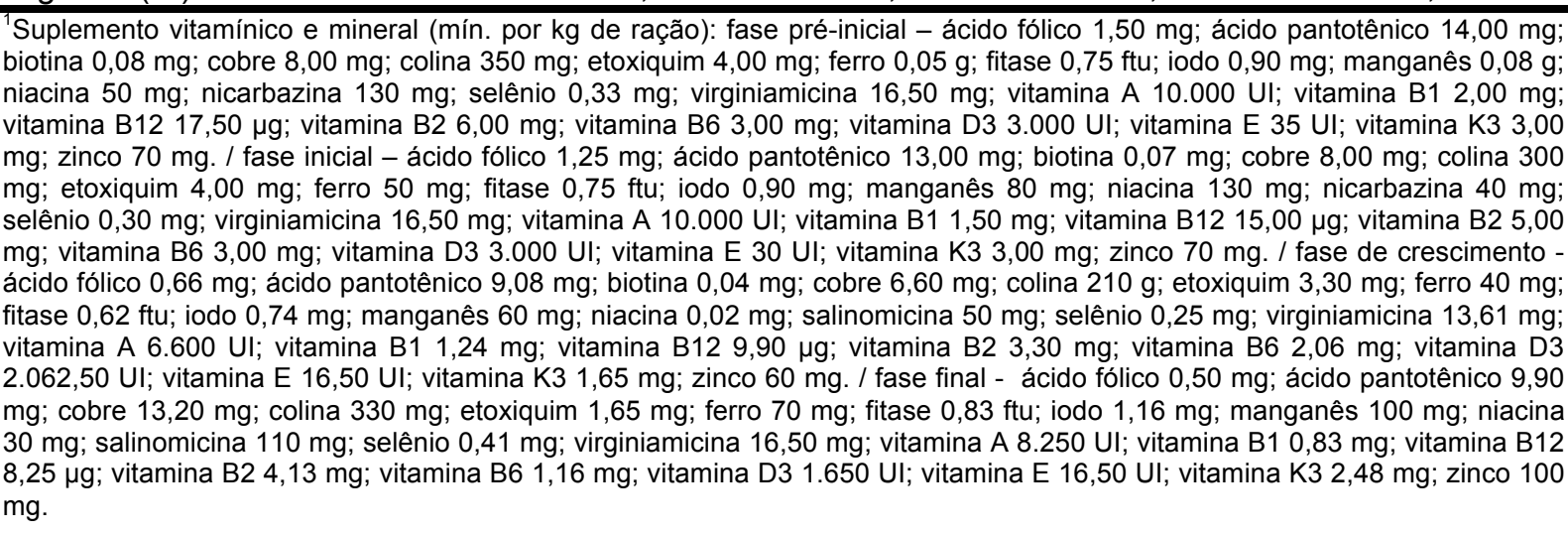 } \\
\hline
\end{tabular}


Foram utilizados 0 antioxidante sintético butil-hidroxi-tolueno $(\mathrm{BHT})$ e o extrato oleoso de urucum, produto comercial fornecido pela empresa KRATOS Indústria e Comércio de Aditivos (Tabela 2). O extrato oleoso de urucum é o produto final da extração da bixina em óleo de soja, e contém $7 \%$ desse carotenoide. A adição nas dietas foi realizada em substituição ao óleo de soja, para que a porcentagem de óleo de soja presente às dietas fosse sempre a mesma, independentemente do tratamento, evitando interferência nos resultados. $\mathrm{O} \mathrm{BHT}$ foi adicionado às dietas de controle positivo em uma concentração de 150 mg/kg de ração.

Tabela 2. Laudo de Análise. Suspensão concentrada de urucum.

\begin{tabular}{|c|c|}
\hline Produto & $\begin{array}{c}\text { Suspensão oleosa de urucum para fins } \\
\text { alimentícios }\end{array}$ \\
\hline \multicolumn{2}{|c|}{ Atributos de Qualidade } \\
\hline $\begin{array}{l}\text { Aspecto } \\
\text { Cor } \\
\text { Odor }\end{array}$ & $\begin{array}{c}\text { Líquido escuro } \\
\text { Avermelhado } \\
\text { Característico do urucum }\end{array}$ \\
\hline \multicolumn{2}{|c|}{ Análise Físico-Química } \\
\hline $\begin{array}{l}\text { Teor de bixina } \\
\text { Densidade } \\
\text { pH direto }\end{array}$ & $\begin{array}{c}7,00 \% \\
0,96 \mathrm{~g} / \mathrm{mL} \\
2,10\end{array}$ \\
\hline \multicolumn{2}{|c|}{ Microbiologia } \\
\hline Contagem total, máx. (UFC/g) & 1000.000 \\
\hline Coliformes totais, máx. (NMP/g) & 100 \\
\hline Coliformes fecais / E.coli & Ausência em $1 \mathrm{~g}$ \\
\hline Bolores / Leveduras, máx (UFC/g) & 1.000 \\
\hline Staphylococcus aureus & Ausência em $0,01 \mathrm{~g}$ \\
\hline Salmonella sp. & Ausência em $25 \mathrm{~g}$ \\
\hline
\end{tabular}

O produto atende a legislação vigente do Ministério da Saúde - Resolução 12/2001 - ANVISA.

O lote de óleo de soja degomado adquirido foi dividido, sendo parte foi submetida à oxidação e parte mantida visando preservar a qualidade. A oxidação do óleo de soja bruto, sem adição de antioxidantes, foi induzida por aquecimento intermitente por 12 horas por dia com temperatura em torno de 80 a $90^{\circ} \mathrm{C}$ em fritadeira elétrica, com constante aeração por um período de 37 dias (Racanicci et al., 2004). O óleo de soja fresco foi mantido fora do alcance de luz e calor e, ao final do período, uma amostra foi coletada para confirmação da qualidade.

Durante o período de oxidação do óleo, foram retiradas amostras periódicas para quantificação de produtos da oxidação (dienos e trienos) presentes no óleo, visando acompanhar o estado oxidativo do ingrediente. O método utilizado foi descrito por Dieffenbacher e Pocklington, (1992), que determina a absorbância do óleo no espectro ultravioleta e fornece seu grau de deterioração dos lipídios decorrente da oxidação lipídica. A formação de dienos conjugados está relacionada ao início do processo de oxidação de óleos (hidroperóxidos e radicais peróxidos). Enquanto que à formação de compostos secundários de estruturas mais estáveis, os trienos conjugados (cetonas insaturadas e aldeídos).

Ainda, antes do início da oxidação e ao final do processo, foram realizadas análises de nível de acidez e índice de peróxidos do óleo. O índice de peróxido (IP) é um método clássico e sensível na determinação de hidroperóxidos, produtos primários da oxidação. Expresso como o número de miliequivalentes de oxigênio ativo (ou peróxido) por 1000 gramas de gordura, foi determinado de acordo com 0 método cd 8-53 da AOCS (2004). O 
índice de acidez pode ser expresso em índice de acidez por cento ou em g do componente ácido principal. É definido como o número de $\mathrm{mg}$ de hidróxido de potássio $(\mathrm{KOH})$ necessário para neutralizar um grama da amostra. O método utilizado foi o $\mathrm{cd} 1 \mathrm{c}-85$ da AOCS (2004), aplicável à óleos brutos e refinados.

O consumo de ração (CR), o ganho de peso (GP) e a conversão alimentar (CA) foram calculados ao final de cada fase de criação $(7,21$ e 42 dias de idade). O CR foi determinado por meio da diferença entre a quantidade de ração fornecida no início e as sobras existentes ao final de cada período, e o resultado obtido foi dividido pelo número médio de aves de cada boxe. O GP médio foi calculado pela diferença entre o peso total das aves de cada boxe no início e ao final de cada fase de criação. CA foi calculada pela divisão entre o total de ração fornecida e o total de ganho de peso no período, e corrigida pelo peso das aves mortas. A mortalidade das aves nas parcelas experimentais foi anotada diariamente, para cálculo da viabilidade (VB). O fator eficiência produtiva (FEP) foi calculado aos 42 dias de idade, utilizando-se a fórmula:

$$
\mathrm{FEP}=\left(\frac{\mathrm{GPM} \times \mathrm{VB}}{\mathrm{CA}}\right) / 10
$$

Em que: GPM = Ganho de peso médio diário (em gramas).

Aos 42 dias de idade, cinco aves por boxe (aves por tratamento) foram abatidas aleatoriamente por sangria após insensibilização elétrica (Geave, São Paulo, Brasil, regulado com voltagem de $100 \mathrm{~mA}$ e frequencia de $600 \mathrm{~Hz}$ ), depenadas e evisceradas para avaliações de qualidade da carne. Peito e coxas foram coletados e os peitos e coxas foram desossados e mantidos refrigerados $\left(4^{\circ} \mathrm{C}\right)$. Com 24 horas post- mortem, o $\mathrm{pH}$, a cor, a perda de água por exsudação (PPE), perda por cocção (PPC) e a força de cisalhamento (FC) foram avaliados em amostras de peito. Para a análise sensorial de aroma estranho (ranço), essas amostras foram congeladas em freezer $-20^{\circ} \mathrm{C}$ por cinco meses.

Os valores de $\mathrm{pH}$ foram mensurados utilizando-se pHmêtro (Homis, model 238, São Paulo, Brasil) acoplado a uma sonda (Digimed, model CF1, Campo Grande, Brasil) diretamente no músculo Pectoralis major. A cor foi determinada, em triplicata, na superfície ventral do peito, utilizando-se o espectrofotômetro portátil (Minolta, CR 400, New Jersey, USA), no sistema CIELab, no qual foram avaliados os parâmetros $L^{*}$ (luminosidade), $a^{*}$ (teor de vermelho) e $b^{*}$ (teor de amarelo) de acordo com metodologia proposta por Van Laack et al. (2000).

Para determinação da PPE foram utilizadas amostras de aproximadamente 80 gramas do músculo Pectoralis major, conforme metodologia descrita por Rasmussen e Anderson (1996). A determinação da porcentagem de perda por exsudação foi realizada pela diferença entre o peso final e peso inicial da amostra conforme a seguinte equação: \%PE $=(P f-P i) x$ $100 / \mathrm{Pi}$, sendo que $\mathrm{PE}=$ perda de exsudato; $\mathrm{Pf}=$ peso final da amostra; $\mathrm{Pi}$ = peso inicial da amostra.

A determinação da PPC foi realizada em amostras de filés de peito de acordo com o descrito por Honikel (1998). A diferença entre o peso inicial (in natura) e final (cozido) correspondeu à perda de peso por cozimento, apresentada em porcentagem.

A FC foi obtida utilizando o texturômetro TAXT plus (Stable Micro Systems, Surrey, UK), equipado com dispositivo RazorBlade. O equipamento foi calibrado com peso padrão de $5,0 \mathrm{~kg}$ e padrão rastreável, a velocidade de 
descida do dispositivo foi de $10 \mathrm{~mm} / \mathrm{s}$ e a análise foi realizada como recomendado por Cavitt et al. (2004).

Para avaliar a oxidação lipídica na carne, após o abate as amostras de carne de peito e coxas com pele das cinco aves abatidas por repetição foram armazenadas em sacos de polietileno e mantidas congeladas $\left(-20^{\circ} \mathrm{C}\right)$. $\mathrm{O}$ modelo utilizado para o ensaio acelerado da oxidação lipídica foi descrito por Racanicci et al. (2004) e o método utilizado para a quantificação de compostos de ranço foi 0 de Substâncias Reativas ao Ácido Tiobarbitúrico (TBARS), conforme descrito por Madsen et al. (1998). A leitura da absorbância foi feita em comprimento de onda de 532 e $600 \mathrm{~nm}$ utilizando um espectofotômetro (Gehaka, modelo UV-400, São Paulo, Brasil), e a diferença $\left(A_{532} \mathrm{~nm}-A_{600 \mathrm{~nm}}\right)$ utilizada como valor de correção para a turbidez. Os resultados foram expressos em micromoles de malonaldeído (MDA) por quilo de carne, utilizando uma curva padrão $(0,1$ - 6,0nM) preparada com 1,1,3,3-tetraetoxipropano (Merck).
Os dados foram submetidos à análise de variância (ANOVA). As médias do arranjo fatorial (tratamentos 1 a 6) foram comparadas entre si pelo teste de Tukey $(\alpha=5 \%)$. Posteriormente, o fatorial foi comparado com os tratamentos definidos como controle positivo (tratamentos 7 e 8) utilizando o teste de Dunnet $(\alpha=5 \%)$. Foi utilizado o programa estatístico Minitab 16 (Minitab Inc., StateCollege, Estados Unidos).

\section{RESULTADOS E DISCUSSÃO}

O processo de oxidação do óleo foi eficiente uma vez que ao final do período, o óleo oxidado apresentou níveis de peróxido, dienos (absortividade a 232nm) e trienos (absortividade a 268nm) muito mळis elevados (Tabela 3). Segundo Warner et al. (1989), o óleo de soja é considerado oxidado com índice de peróxido de 20 $\mathrm{meq} / \mathrm{kg}$, enquanto que o óleo de girassol apresenta forte aroma com índice de peróxido de 8 e aroma estranho com índice de peróxido de $13 \mathrm{meq} / \mathrm{kg}$.

Tabela 3. Parâmetros analíticos do óleo utilizado nas rações experimentais

\begin{tabular}{lccc}
\hline Parâmetros & $\begin{array}{c}\text { Óleo degomado } \\
\text { (Inicial) }\end{array}$ & $\begin{array}{c}\text { Óleo degomado } \\
\text { (Final 37 dias) }\end{array}$ & $\begin{array}{c}\text { Óleo oxidado } \\
\text { (37dias) }\end{array}$ \\
\hline Índice de acidez $(\mathrm{mg} \mathrm{KOH} / \mathrm{g}$ de óleo) & 1,32 & 1,83 & 1,55 \\
AGL (\% ácido oleico) & 0,66 & 0,92 & 0,78 \\
Índice de peróxido (mEq $\mathrm{O}_{2} / \mathrm{kg}$ de óleo) & $<2,00$ & 10,88 & 51,67 \\
Absortividade a $232 \mathrm{~nm}$ (dienos) & 2,573 & 4,434 & 14,684 \\
Absortividade a $268 \mathrm{~nm}$ (trienos) & 0,259 & 0,700 & 2,290 \\
\hline
\end{tabular}

${ }^{1}$ Ácidos graxos livres.

Os resultados de desempenho estão apresentados na tabela 4. Não houve interação entre a bixina e a qualidade do óleo utilizado na ração.

$A$ adição de bixina na dieta das aves melhorou a CA $(P<0,01)$ e diminuiu - CRM $(P<0,05)$. Outros estudos avaliaram o uso do extrato oleoso de urucum na dieta de frangos de corte e poedeiras comerciais, e mostraram resultados de desempenho diferentes. Silva et al. (2005) recomendam a inclusão do extrato na ração de frangos de corte de 1 a 47 dias em até $5 \%$ e Silva et al. (2006) recomendaram até $12 \%$ de inclusão do extrato em dietas de poedeiras.

O uso de óleo de soja oxidado apresentou efeito negativo na CA $(P<0,01)$, o que era esperado, uma vez que o consume de alimentos oxidados causa prejuízo no desempenho (Lin et al., 1989; Sheehy et al., 1994). Estes resultados estão de acordo com aqueles 
obtidos por Anjum et al. (2004), que concluíram que frangos de corte que receberam ração com $2 \%$ de óleo de soja fresco (3 meq/ $\mathrm{kg}$ ) apresentaram melhor ganho de peso e conversão alimentar do que aqueles alimentados com ração com óleo oxidado (50 $\mathrm{meq} / \mathrm{kg}$ ).
Resultados divergentes foram apresentados por Racanicci et al. (2008) que, em um estudo avaliando 0 desempenho de frangos de corte alimentados com dietas formuladas com óleo de vísceras frescas ou oxidadas (inclusão de 4\%), não observaram efeitos.

Tabela 4. Efeitos de níveis de bixina no desempenho de frangos de corte Ross 308 de 1 a 42 dias de idade.

\begin{tabular}{|c|c|c|c|c|c|c|}
\hline$\%$ BIXINA & ÓLEO & $\mathbf{G P M}^{1}$ & $\mathrm{CRM}^{2}$ & $\mathrm{CA}^{3}$ & VIAB. $^{4}$ & FEP $^{5}$ \\
\hline & & \multicolumn{2}{|c|}{---------------(g)----------- } & \multicolumn{3}{|c|}{$(\%)$} \\
\hline 0,00 & - & 2866,9 & $4982,5^{A}$ & $1,77^{\mathrm{A}}$ & 94,0 & 372,6 \\
\hline 0,05 & - & 2869,8 & $4753,2^{C}$ & $1,68^{\mathrm{B}}$ & 91,2 & 380,4 \\
\hline 0,10 & - & 2889,0 & $4865,8^{\mathrm{B}}$ & $1,71^{\mathrm{B}}$ & 94,5 & 390,9 \\
\hline- & Fresco & 2906,8 & 4851,4 & $1,69^{A}$ & 92,8 & 388,6 \\
\hline- & Oxidado & 2843,6 & 4882,9 & $1,74^{\mathrm{B}}$ & 93,6 & 373,9 \\
\hline 0,00 & Fresco & 2940,7 & 4974,3 & 1,73 & 92,0 & 380,9 \\
\hline 0,00 & Oxidado & 2793,1 & 4990,6 & 1,80 & 96,0 & 364,2 \\
\hline 0,05 & Fresco & 2892,3 & 4729,2 & 1,66 & 89,6 & 381,4 \\
\hline 0,05 & Oxidado & 2847,3 & 4777,2 & 1,70 & 92,8 & 379,4 \\
\hline 0,10 & Fresco & 2887,5 & 4850,7 & 1,69 & 96,8 & 403,6 \\
\hline 0,10 & Oxidado & 2890,5 & 4880,9 & 1,72 & 92,1 & 378,2 \\
\hline $0,00+\mathrm{BHT}$ & Fresco & 2899,8 & 4909,6 & 1,71 & 96,8 & 401,7 \\
\hline $0,00+\mathrm{BHT}$ & Oxidado & 2901,7 & 4907,2 & 1,72 & 90,4 & 373,3 \\
\hline CV (\%) & & 3,15 & 3,54 & 3,44 & 6,60 & 8,74 \\
\hline \multicolumn{7}{|l|}{ Probabilidade } \\
\hline Bixina & & 0,853 & 0,024 & 0,004 & 0,405 & 0,471 \\
\hline Óleo & & 0,081 & 0,624 & 0,024 & 0,688 & 0,234 \\
\hline Bixina*Óleo & & 0,217 & 0,979 & 0,800 & 0,201 & 0,729 \\
\hline Controles & & 0,966 & 0,979 & 0,658 & 0,195 & 0,266 \\
\hline Controle*Fatorial & & 0,449 & 0,520 & 0,807 & 0,888 & 0,618 \\
\hline
\end{tabular}

Na tabela 5 estão apresentados os resultados de rendimento de carcaça, peito, coxa e porcentagem de gordura abdominal dos frangos de corte, abatidos aos 42 dias de idade. Não houve efeito de tratamento sobre o rendimento de carcaça e peito.

Observou-se que a utilização de óleo de soja fresco na dieta reduziu $(P<0,01)$ a quantidade de gordura abdominal em relação às aves alimentadas que receberam óleo de soja oxidado. A inclusão de bixina na dieta também reduziu a quantidade de gordura abdominal $(\mathrm{P}<0,01)$. Este resultado é relevante em termos de mercado, uma vez que esta gordura não é apreciada pelo consumidor e, segundo Carter (1993), o novo perfil da taxa de crescimento do frango de linhagens de crescimento acelerado que resultou na elevação da taxa metabólica no início do ciclo produtivo, tem sido relatada como a maior responsável por um maior acúmulo de gordura na carcaça dos frangos de corte. Sendo assim, a ação da bixina no metabolismo dos frangos merece maiores estudos. Esses resultados diferem dos encontrados por Silva et al. (2005) que, em trabalho realizado com a inclusão do extrato oleoso de urucum na dieta de frangos de corte, não observaram efeitos sobre a porcentagem de gordura abdominal.

Nas variáveis de qualidade de carne, observou-se efeito da bixina nos teores de amarelo $(P<0,01)$ e vermelho $(P<0,01)$ da carne de peito, que aumentaram linearmente com 0 aumento dos níveis adicionados às 
dietas (Tabela 6). Segundo Harder et al. (2010), o urucum pode ser utilizado como agente pigmentante para melhorar a coloração dos cortes de carne de frangos. A cor da carne e da pele é uma característica importante que influencia diretamente sua aceitabilidade pelo consumidor no momento da compra, porém não há valores considerados padrão para a carne de frango.

Tabela 5. Efeitos de níveis de bixina no rendimento de carcaça, peito, coxa e porcentagem de gordura abdominal de frangos de corte aos 42 dias de idade.

\begin{tabular}{|c|c|c|c|c|c|}
\hline$\%$ BIXINA & ÓLEO & Carcaça & Peito & Coxas & Gordura Abdominal \\
\hline & & & & $---(\%)--$ & --------" \\
\hline 0,00 & - & 73,3 & 36,5 & 30,5 & $1,7^{\mathrm{A}}$ \\
\hline 0,05 & - & 74,0 & 37,0 & 30,4 & $1,4^{\mathrm{B}}$ \\
\hline 0,10 & - & 73,3 & 37,5 & 29,8 & $1,4^{\mathrm{B}}$ \\
\hline- & Fresco & 73,7 & 37,2 & 30,05 & $1,4^{\mathrm{B}}$ \\
\hline- & Oxidado & 73,4 & 36,7 & 30,42 & $1,6^{\mathrm{A}}$ \\
\hline 0,00 & Fresco & 74,0 & 36,9 & $29,63^{c}$ & 1,47 \\
\hline 0,00 & Oxidado & 72,6 & 36,1 & $31,40^{a}$ & 1,87 \\
\hline 0,05 & Fresco & 73,4 & 37,2 & $30,62^{\mathrm{b}}$ & 1,32 \\
\hline 0,05 & Oxidado & 74,5 & 36,7 & $30,22^{b}$ & 1,53 \\
\hline 0,10 & Fresco & 73,6 & 37,6 & $29,91^{\text {bc }}$ & 1,33 \\
\hline 0,10 & Oxidado & 73,0 & 37,3 & $29,65^{c}$ & 1,43 \\
\hline $0,00+\mathrm{BHT}$ & Fresco & 73,4 & 37,1 & 30,45 & 1,44 \\
\hline $0,00+\mathrm{BHT}$ & Oxidado & 73,1 & 36,8 & 29,44 & 1,26 \\
\hline CV (\%) & & 6,20 & 8,04 & 6,78 & 30,61 \\
\hline \multicolumn{6}{|l|}{ Probabilidade } \\
\hline Bixina & & 0,774 & 0,356 & 0,219 & 0,007 \\
\hline Óleo & & 0,720 & 0,332 & 0,323 & 0,003 \\
\hline Bixina*Óleo & & 0,498 & 0,950 & 0,037 & 0,300 \\
\hline Controles & & 0,753 & 0,927 & 0,111 & 0,179 \\
\hline Controle* Fatorial & & 0,805 & 0,741 & 0,455 & 0,085 \\
\hline
\end{tabular}

Nas variáveis de qualidade de carne, observou-se efeito da bixina nos teores de amarelo $(P<0,01)$ e vermelho $(P<0,01)$ da carne de peito, que aumentaram linearmente com 0 aumento dos níveis adicionados às dietas (Tabela 6). Segundo Harder et al. (2010), o urucum pode ser utilizado como agente pigmentante para melhorar a coloração dos cortes de carne de frangos. A cor da carne e da pele é uma característica importante que influencia diretamente sua aceitabilidade pelo consumidor no momento da compra, porém não há valores considerados padrão para a carne de frango.

Não houve efeito de tratamento para as demais características de qualidade. Os valores de $\mathrm{pH}$ das amostras nesse experimento variaram de 5,8 a 5,9. Segundo Venturini et al. (2007), o pH da carne de frango diminui visto à formação de ácido lático e a carne de peito deve apresentar $\mathrm{pH}$ final entre 5,7 e 5,9. Após 24 horas, se o pH estiver superior a 6,2, a carne de frango irá apresentar grande retenção de água, o que implica em curto tempo de conservação e o estabelecimento da coloração escura, caracterizando a carne DFD (dark, firm and dry). Caso o $\mathrm{pH}$ se encontre abaixo de 5,8 em menos de 4 horas, teremos a carne PSE (pale, soft, exudative) caracterizado pela má retenção de água além do aspecto pálido e mole.

Os resultados as análises de oxidação lipídica nas carnes do peito e da coxa das aves estão apresentados nas tabelas 7 e 8 , respectivamente.

Para a carne de peito crua (Tabela 7), os tratamentos sem adição de bixina apresentaram maiores valores de TBARs $(P<0,1)$ em comparação com os 
tratamentos com BHT, indicando maior oxidação. Esses resultados comprovam a ação antioxidante do BHT, independente da qualidade do óleo utilizado. O tratamento com adição de $0,05 \%$ de bixina e óleo de soja fresco não diferiu dos tratamentos controle positivos, com BHT, demonstrando ação antioxidante do carotenoide. Porém, quando o óleo de soja oxidado foi utilizado, esse nível de bixina não foi suficiente para controlar a oxidação das amostras.

Tabela 6. Efeitos de níveis de bixina sobre os parâmetros de qualidade de carne do peito.

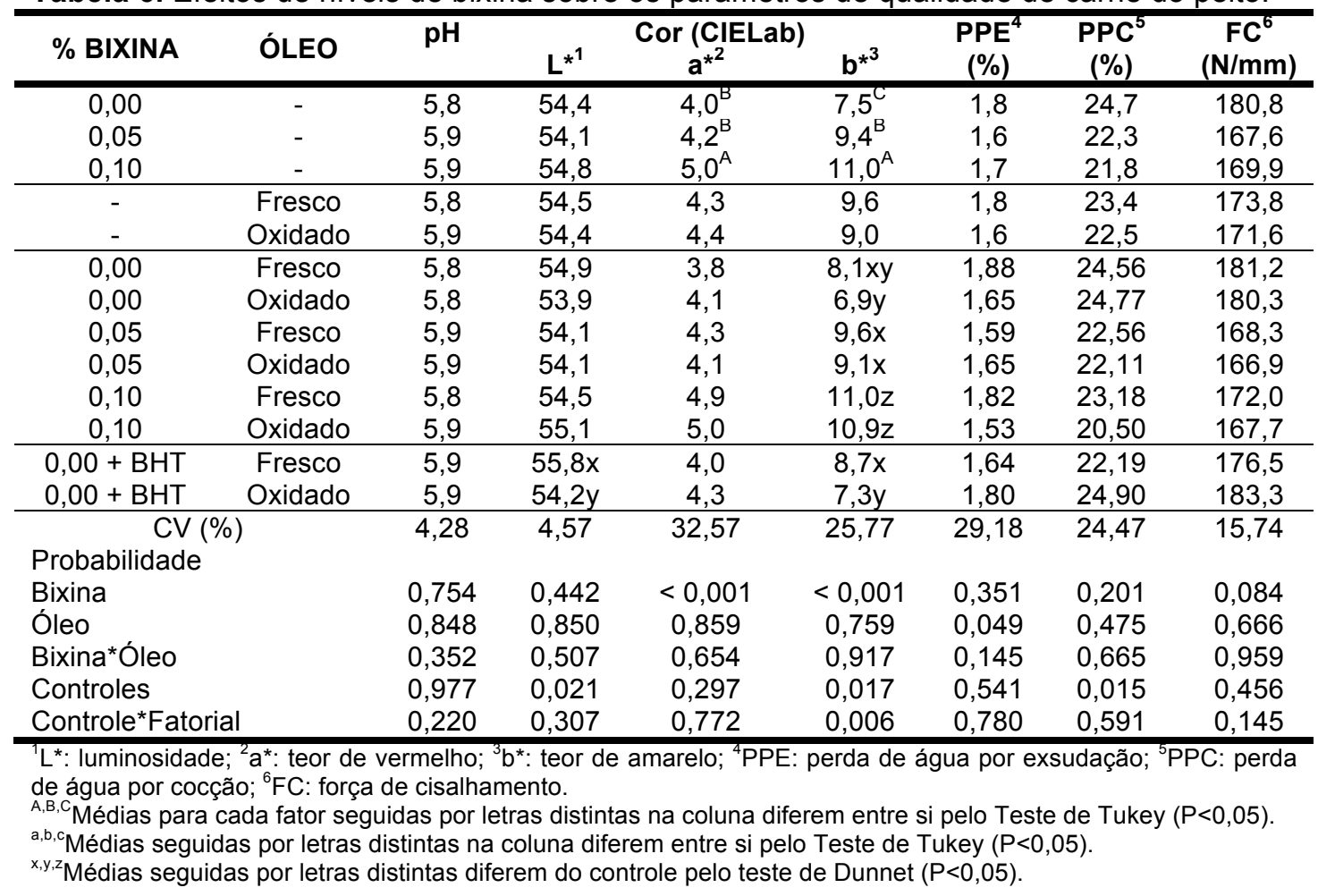

Ainda na carne de peito crua, observou-se que o tratamento com adição de $0,10 \%$ de bixina e óleo de soja fresco não apresentou melhor efeito antioxidante do que aquele formulado com $0,05 \%$ de bixina, o que pode ser explicado pela atividade próoxidante apresentada por este tratamento. A atividade pró-oxidante dos carotenóides está diretamente relacionada ao potencial redox da molécula e ao ambiente celular em que ela atua. Além disso, pode ser influenciado pela quantidade de carotenóides administrados - em altas doses, pode acelerar a taxa de oxidação ao invés de reduzir - e pela presença de outros antioxidantes (Palozza, 1998). Neste estudo, para a carne do peito, a inclusão de $0,10 \%$ de bixina parece ser muito elevada, o que provavelmente provocou um desequilíbrio entre os componentes oxidantes e antioxidantes existentes no modelo cárneo, levando a um aumento na formação de radicais em níveis elevados e difíceis de reparar pelo antioxidante (Young e Lowe, 2001), resultando em aumento do TBARS (efeito pró-oxidante).

Após 0 tratamento térmico, observou-se que, para dietas formuladas com óleo de soja fresco, a inclusão de $0,10 \%$ de bixina controlou a oxidação tão bem quanto o antioxidante sintético BHT $(P=0,021)$. Sabe-se que o tratamento térmico favorece 0 processo oxidativo (Lima Junior et al., 2013), uma vez que leva à liberação de oxigênio ferro, induzindo a produção de radicais livres (Byrne et al., 2002) o que 
explica o resultado um pouco mais elevado apresentado pelo tratamento com $0,05 \%$ de bixina, e, diante dessa situação, foi necessária a adição de maior quantidade de antioxidante natural.

Três dias após o cozimento, não houve diferença entre os tratamentos. Os tratamentos com óleo oxidado tiveram valores de TBARS mais altos, o que era esperado. Aos 10 dias após o cozimento das amostras, a adição de $0,05 \%$ de bixina na dieta controlou a oxidação $(P>0,01)$ eficientemente. $O$ tratamento com inclusão de $0,05 \%$ de bixina em óleo de soja oxidado apresentou os melhores resultados $(P>0,05)$, controlando a oxidação de maneira similar ao antioxidante sintético (BHT).

$\mathrm{Na}$ carne de peito cozida, da mesma forma que na carne de peito crua, o melhor controle da oxidação das amostras no tratamento com menor inclusão de bixina na dieta $(0,05 \%)$ pode ter ocorrido em virtude da atividade próoxidante apresentada pelo carotenoide bixina, possivelmente presente em excesso no tratamento contendo $0,10 \%$.

Para a carne de coxa crua, não houve diferença entre os tratamentos. Após 0 tratamento térmico das amostras, níveis mais elevados de bixina na dieta controlaram de maneira mais eficaz a oxidação das amostras $(P<0,01)$, independentemente da qualidade do óleo utilizado. Isso pode ter acontecido devido ao maior potencial oxidativo da carne de coxa em relação à do peito, uma vez que a maior presença de gordura, além da circulação sanguínea e da maior presença de ferro e outros agentes oxidantes neste músculo aceleram a oxidação lipídica (Dransfield e Sosnicki, 1999; Fellenberg e Speisk, 2006; Lu, et al., 2010; TACO, 2011), exigindo maiores dosagens de antioxidantes para a proteção dos lipídios.
O potencial oxidativo da carne pode variar dependendo da espécie animal, tipo de músculo, localização anatômica e composição lipídica. Os ácidos graxos poli-insaturados, mais passiveis de sofrerem oxidação, estão em maior quantidade no musculo do peito quando comparados ao músculo da coxa e sobrecoxa. Em contrapartida, por apresentar maior conteúdo e maior variedade de tipos de lipídios, a ocorrência da oxidação se torna mais rápida na carne da coxa e sobrecoxa, em relação a carne do peito (Dransfield e Sosnicki, 1999).

Nas poedeiras e frangos de corte, o músculo peitoral, de cor branca, tem predominantemente fibras FG (tipo IIB, de contração rápida e glicolítica) e FOG (tipo IIA, contração rápida e oxidativa) e, histologicamente, pequena densidade de capilares sanguíneos e pequeno número de mitocôndrias. Os músculos vermelhos, da coxa e sobrecoxa, por outro lado, são ricamente vascularizados e com grande número de mitocôndrias em suas fibras, principalmente do tipo SO (tipo I, contração lenta e oxidativa) e FOG (MACARI et al., 2002). Essa diferença na composição também resulta em maior oxidação lipídica na carne da coxa em relação a carne do peito, uma vez que o ferro liberado da mioglobina reage com os ácidos graxos poliinsaturados, resultando na geração de radicais livres e na propagação de reações oxidativas (Fellenberg \& Speisk, 2006; Lu, et al., 2010).

Dez dias após o tratamento térmico das amostras, o tratamento com adição de $0,05 \%$ de bixina e óleo oxidado apresentou o melhor resultado, ou seja, menor valor de TBARS $(P<0,05)$, e não diferiu do tratamento com BHT, indicando efeito antioxidante da bixina. 
Tabela 7. Valores médios de substâncias reativas ao ácido tiobarbitúrico ( $\mu \mathrm{mol}$ de MDA $/ \mathrm{kg}$ de amostra) na carne de peito crua e após cozimento (dia 0) e armazenada sob refrigeração $\left(4^{\circ} \mathrm{C}\right)$ por até 10 dias.

\begin{tabular}{|c|c|c|c|c|c|c|}
\hline \multirow{2}{*}{$\%$ BIXINA } & \multirow{2}{*}{ ÓLEO } & \multirow[b]{2}{*}{ Crua } & & \multicolumn{2}{|c|}{ Após cozimento } & \multirow[b]{2}{*}{ Dia 10} \\
\hline & & & Dia 0 & Dia 3 & Dia 7 & \\
\hline & & \multicolumn{5}{|c|}{ 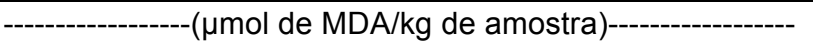 } \\
\hline 0,00 & - & 0,8 & 5,5 & 23,9 & 35,9 & $49,0^{\mathrm{A}}$ \\
\hline 0,05 & - & 0,7 & 4,1 & 22,0 & 34,1 & $43,0^{\mathrm{B}}$ \\
\hline 0,10 & - & 0,8 & 3,9 & 26,6 & 39,4 & $52,3^{\mathrm{A}}$ \\
\hline- & Fresco & 0,7 & 4,3 & $22,1^{\mathrm{B}}$ & 36,6 & 49,4 \\
\hline- & Oxidado & 0,8 & 4,7 & $26,2^{\mathrm{A}}$ & 36,3 & 46,8 \\
\hline 0,00 & Fresco & $0,75 y$ & $6,39^{a} z$ & 24,32 & $38,53^{\mathrm{ab}} y$ & $49,02 x y$ \\
\hline 0,00 & Oxidado & $0,75 y$ & $4,59^{b} x$ & 23,55 & $33,33^{b} z$ & $48,95 x y$ \\
\hline 0,05 & Fresco & $0,62 x$ & $3,81^{c} x$ & 20,20 & $33,75^{\mathrm{b}} \mathrm{z}$ & $44,91 \mathrm{y}$ \\
\hline 0,05 & Oxidado & $0,77 y$ & $4,32^{\mathrm{bc} x}$ & 23,73 & $34,52^{\mathrm{b}} \mathrm{y}$ & $41,08 z$ \\
\hline 0,10 & Fresco & $0,76 y$ & $2,79^{d} y$ & 21,79 & $37,60^{a b} y$ & $54,31 x y$ \\
\hline 0,10 & Oxidado & $0,77 y$ & $5,05^{\mathrm{b}} \mathrm{z}$ & 31,42 & $41,10^{a} x$ & $50,33 x y$ \\
\hline $0,00+\mathrm{BHT}$ & Fresco & $0,57 x$ & $2,82 y$ & $21,88 y$ & $39,11 y$ & $50,51 y$ \\
\hline $0,00+\mathrm{BHT}$ & Oxidado & $0,61 x$ & $4,20 x$ & $33,60 x$ & $44,20 x$ & $56,22 x$ \\
\hline CV (\%) & & 16,20 & 27,64 & 24,92 & 10,77 & 10,92 \\
\hline \multicolumn{7}{|l|}{ Probabilidade } \\
\hline Bixina & & 0,333 & 0,001 & 0,131 & 0,022 & 0,003 \\
\hline Óleo & & 0,360 & 0,014 & 0,019 & 0,572 & 0,147 \\
\hline Bixina*Óleo & & 0,719 & 0,001 & 0,106 & 0,040 & 0,555 \\
\hline Controles & & 0,714 & 0,002 & 0,022 & 0,045 & 0,048 \\
\hline Controle*Fatorial & & 0,001 & 0,021 & 0,171 & 0,003 & 0,038 \\
\hline
\end{tabular}

Tabela 8. Valores médios de substâncias reativas ao ácido tiobarbitúrico ( $\mu$ mol de MDA $/ \mathrm{kg}$ de amostra) na carne da coxa crua e após cozimento (dia 0) e armazenada sob refrigeração $\left(4^{\circ} \mathrm{C}\right)$ por até 10 dias.

\begin{tabular}{|c|c|c|c|c|c|c|}
\hline \multirow{2}{*}{$\%$ BIXINA } & \multirow{2}{*}{ ÓLEO } & \multirow[b]{2}{*}{ Crua } & \multicolumn{4}{|c|}{ Após cozimento } \\
\hline & & & Dia 0 & Dia 3 & Dia 7 & Dia 10 \\
\hline \multicolumn{7}{|c|}{-----------------------( $(\mu \mathrm{mol}$ de MDA/kg de amostra)-------------- } \\
\hline 0,00 & - & 1,1 & 11,5 & 46,7 & 60,7 & $92,7^{\mathrm{AB}}$ \\
\hline 0,05 & - & 1,2 & 10,1 & 50,5 & 65,4 & $90,6^{\mathrm{B}}$ \\
\hline 0,10 & - & 1,1 & 6,7 & 51,2 & 59,3 & $98,3^{\mathrm{A}}$ \\
\hline- & Fresco & 1,1 & 9,5 & 48,1 & 64,4 & 93,3 \\
\hline - & Oxidado & 1,2 & 9,3 & 50,8 & 59,2 & 94,4 \\
\hline 0,00 & Fresco & $1,19 x$ & $11,05^{\mathrm{ab}} \mathrm{y}$ & 45,48 & $59,24^{\mathrm{ab}} x y$ & $91,94 x$ \\
\hline 0,00 & Oxidado & $1,03 x y$ & $12,03^{a} y$ & 47,88 & $62,08^{a b} x y$ & $93,37 x$ \\
\hline 0,05 & Fresco & $1,00 x y$ & $10,17^{\mathrm{b}} \mathrm{y}$ & 48,10 & $67,16^{a} x$ & $93,15 x$ \\
\hline 0,05 & Oxidado & $1,35 x$ & $9,93^{b} y$ & 52,90 & $63,70^{a} x y$ & $88,01 x y$ \\
\hline 0,10 & Fresco & $1,13 x$ & $7,33^{\mathrm{c} y}$ & 50,82 & $66,82^{a} x$ & $94,75 x$ \\
\hline 0,10 & Oxidado & $1,10 x y$ & $6,03^{c} y$ & 51,60 & $51,84^{b} y$ & $101,83 z$ \\
\hline $0,00+\mathrm{BHT}$ & Fresco & $1,13 x$ & $4,41 x$ & 44,37 & $55,30 y$ & $81,24 y$ \\
\hline $0,00+\mathrm{BHT}$ & Oxidado & $0,88 y$ & $3,60 x$ & 45,27 & $63,16 x y$ & $86,40 x$ \\
\hline CV $(\%)$ & & 19,44 & 37,23 & 8,65 & 10,69 & 7,69 \\
\hline \multicolumn{7}{|l|}{ Probabilidade } \\
\hline Bixina & & 0,938 & 0,000 & 0,104 & 0,039 & 0,012 \\
\hline Óleo & & 0,758 & 0,511 & 0,146 & 0,013 & 0,566 \\
\hline Bixina*Óleo & & 0,441 & 0,012 & 0,650 & 0,004 & 0,057 \\
\hline Controles & & 0,004 & 0,085 & 0,686 & 0,077 & 0,049 \\
\hline \multirow[t]{2}{*}{ Controle*Fatorial } & & 0,001 & $<$ & 0,020 & 0,001 & 0,001 \\
\hline & & & 0,001 & & & \\
\hline
\end{tabular}




\section{CONCLUSÕES}

A inclusão de bixina na dieta dos frangos de corte melhorou a conversão alimentar e reduziu a gordura abdominal, contudo, o uso desse aditivo e de outros carotenóides na dieta dos frangos de corte exige cautela, uma vez que o excesso pode apresentar efeito negativo na qualidade da carne, aumentando a oxidação lipídica devido ao efeito pró-oxidante, e reduzindo o "shelf-life". O uso de óleo de soja oxidado na dieta de frangos de corte prejudica a conversão alimentar e aumenta a deposição de gordura abdominal. Apesar de terem sido observados efeitos antioxidantes na carne de peito e coxa, a bixina não pode ser recomendada como uma alternativa natural ao antioxidante sintético BHT.

\section{AGRADECIMENTOS}

Este trabalho foi financiado pela Fundação de Amparo à Pesquisa do Estado de São Paulo - FAPESP [números de subsídios 2011 / 23731-5 e 2012 / 05415-1]. Os autores também gostariam de agradecer à KRATOS Indústria e Comércio de Aditivos (CONDITEM Condimentos e Temperos) pela doação do extrato oleoso de urucum.

\section{NOTAS INFORMATIVAS}

Todos os procedimentos utilizados foram aprovados pela Comissão de Ética no Uso de Animais (CEUA) da Faculdade de Medicina Veterinária e Zootecnia - UNESP, Botucatu sob o protocolo número 273/2011.

\section{REFERÊNCIAS}

AOCS,Official Methods and Recommended Practices of the AOCS. 5th Ed., American OilChemist's Society, Champaign, Illinois, USA, 2004.
ANJUM, M.I.; MIRZA, I.H.; KHAN, A.G. et al. Effect of fresh versus oxidized soybean oil on growth performance, organs weights and meat quality of broiler chicks. Pakistan Veterinary Journal, v.24, p.173-178, 2004.

O'BYRNE, D.J.; DEVARAJ, S.; GRUNDY, S.M.; JIALAL, L. Comparison of the antioxidant effects of Concord grape juice flavonoids alpha-tocopherol on markers of oxidative stress in healthy adults. The American Journal of Clinical Nutrition, v.76, n.6, p.13671374, 2002.

CABEL, M.C.; WALDROUP, P.W.; SHERMER, W.D. et al. Effects of ethoxyquin feed preservative and peroxide level on broiler performance. Poultry Science, v.67, p.1725-1730, 1988.

CARTER, I. Evolução genética dos frangos de corte até o século. In: CONGRESSO BRASILEIRO DE AVICULTURA, 21, 1993, Brasília. Anais... Brasília: Fundação APINCO de Ciências e Tecnologia Avícolas, 1993.

CAVITT, L.C.; YOUM, G.W.; MEULLENET, J.F. et al. Prediction of poultry meat tenderness using razor blade shear, allo-kramer shear, and sarcomere length. Journal of Food Science, v.69, SNQ11-SNQ15, 2004.

DIEFFENBACHER, A.; POCKLINGTON, W.D. Standard methods for analysis of oils, fat and derivates. 7.ed. Oxford: International Union of Pure an Applied Chemistry (IUPAC), 1992.

DRANSFIELD, E.; SOSNICKI, A. Relationship between muscle growth and poultry meat quality. Poultry Science, v.78, p.743-746, 1999. 
ENGBERG, R.M.; LAURIDSEN, C.; JENSEN, S.K. et al. Inclusion of oxidized vegetable oil in broiler diets. Its influence on nutrient balance and on the antioxidative status of broilers. Poultry Science, v.75, p.1003-1011, 1996.

FELLENBERG, M. A.; SPEISKY, $\mathrm{H}$. Antioxidants: their effects on broiler oxidative stress and its meat oxidative stability. World Poultry Science Journal, v.62, p.53-70, 2006.

GARCIA C.E.R.; BOLOGNESE, V.J.; DAYS, J.F.G. et al. Carotenoides bixina e norbixina extraídos do urucum (BixaorellanaL.) como antioxidantes em produtos cárneos. Ciência Rural, v.42, p.1510-1517, 2012.

HARDER, M.N.C.; CANNIATTIBRAZACA, S.G.; COELHO, A.A.D.et al. Cholesterol and iron availability in yolk of laying hens feed with annatto (Bixaorellana). Animal, v.1, p.477-482, 2007.

HONIKEL, K.O. Reference methods for the assessment of physical characteristics of meat. Meat Science, v.49, p.447-457, 1998.

LIMA JÚNIOR, D.M.; RANGEL, A.H.N.; URBANO, S.A. et al. 2013. Lipid oxidation and lamb meat quality. Acta Veterinária Brasilica, v.7, p.14-28, 2013.

LIMA, L.R.P.; OLIVEIRA, T.T.; NAGEM, T.J. Efeitos do flavonóide quercetina e dos corantes bixina e norbixina sobre parâmetros sanguíneos de coelhos. Revista de Nutrição, v.16, n.3, p.305314, 2003.

LIN, C.F.; ASGHAR, J.I.; GRAY, A. et al. Effects of oxidized dietary oil and antioxidant supplementation on broiler growth and meat stability. British Poultry Science, v.30, p.855-864, 1989.
LU, J.; LIN, P.H.; YAO, Q.; CHEN, C. Chemical and molecular mechanisms of antioxidants: experimental approaches and model systems. Journal of Cellular and Molecular Medicine, v.14, p.840860, 2010.

MACARI, M.; FURLAN, R.L.; GONZALES, E. Fisiologia aviária aplicada a frangos de corte. 2.ed. Jaboticabal: FUNEP, 2002.

MADSEN, H.L.; SORENSEN, B.; SKIBSTED, L.H. et al. The antioxidative activity of summer savory (SaturejahortemisL.) and rosemary (Rosmarinus officinalis L.) in dressing stored exposed to light or in darkness. Food Chemistry, v.63, p.173-180, 1998.

MINITAB $16 \quad$ STATISTICAL SOFTWARE. [Computer software]. PA: Minitab, Inc. 2010.

NÚCLEO DE ESTUDOS E PESQUISAS EM ALIMENTAÇÃO - TACO. Tabela brasileira de composição de alimentos. 4. ed. Campinas: NEPA / UNICAMP, 2011. 161p.

PALOZZA , P. Prooxidant actions of carotenoids in biologic systems. Nutrition Reviews, v.56, p.257-265, 1998.

RACANICCI, A.M.C.; MENTEN, J.F.M.; REGITANO-D'ARCE, M.A.B. et al. Efeito do uso de óleo de vísceras de aves oxidado na ração de frangos de corte sobre o desempenho, a composição da carcaça e a estabilidade oxidativa da carne da sobrecoxa. Revista Brasileira de Zootecnia, v.37, p.443-449, 2008.

RACANICCI, A.M.C.; MENTEN, J.F.M.; REGIATNO-D'ARCE, M.A.B. et al. Oxidação lipídica do óleo de vísceras de 
aves para redução de seu conteúdo de energia metabolizável para frangos de corte na fase de crescimento. Revista Brasileira de Zootecnia, v.33, n.4, p.919-923, 2004.

RASMUSSEN, A.J.; ANDERSSON, M. New method for determination of drip loss in pork muscles. In: INTERNATIONAL CONGRESS ON MEAT SCIENCE AND TECHNOLOGY, 42, Lillehammer. Proceedings... Lillehammer: Meat for the Consumer, 1996, p. 286-287.

ROSTAGNO, H.S.; ALBINO, L.F T.; DONZELE, $\mathrm{J}$ L.; et al. Tabelas brasileiras para aves e suínos: composição de alimentos e exigências nutricionais de aves e suínos. 3. ed. Viçosa: UFV, 2011. 252 p.

SCOTT, ML. Nutrition of the chicken. 3.ed. Ithaca: Scott, M.L and Associates, 1982.

SHEEHY, P.J.; MORRISSEY, P.A.; FLYNN, A. Consumption of thermally oxidized sunflower oil by chicks reduces a-tocopherol status and increases susceptibility of tissues to lipid oxidation. British Journal of Nutrition, v.71, p.5365, 1994.

SILVA, C.R.; ANTUNES, L.M.; BIANCHI, M.L. Antioxidant action of bixin against cisplatin-induced chromosome aberrations and lipid peroxidation in rats. Pharmacologycal Research, v.43, p.561-566, 2001.

SILVA, J.H.V.; SILVA, E.L.; JORDÃO FILHO, J. et al. Efeitos da inclusão do resíduo da semente de urucum (Bixa orellana L.) na dieta para frangos de corte: desempenho e características de carcaça. Brazilian Journal of Animal Science, v.34, p.1606-1613, 2005.
SILVA, J.H.V.; SILVA, E.L.; JORDÃO FILHO, J. et al. Urucum (Bixa orellana L.) como corante da gema, pele, bico e ovário de poedeiras avaliado por dois métodos analíticos. Ciência e Agrotecnologia, v.30, p.988-994, 2006.

VAN LAACK, R.L.; LIU, C.H.; SMITH, M.O. et al. Characteristics of pale, soft, exudative broiler breast meat. Poultry Science, v.79, p.1057-1061, 2000.

VENTURINI, K.S.; SARCINELLI, M.F.; SILVA, L.C. Características da Carne de Frango. Boletim Técnico. Universidade Federal do Espírito Santo - UFES, 2007. Disponível em: < http://www.agais.com/telomc/b01307_ca racteristicas_carnefrango.pdf> Acesso em: 09/01/2017.

WARNER, K.; FRANKEL, E.N.; MOUNTS, T.L. Flavor and oxidative stability of soybean, sunflower and low erucic acid rapeseed oils. Journal of the American Oil Chemists' Society, v.66, p.558-564, 1989.

YOUNG, A.J.; LOWE, G.M. Antioxidant and Prooxidant Properties of Carotenoids. Archives of Biochemistry and Biophysics, v.385, p.20-27, 2001.

ZHENG, W.; WANG, S.Y. Antioxidant activity and phenolic compounds in selected herbs. Journal of Agricultural and Food Chemistry, v.49, p.51655170, 2001. 